Chapman University

Chapman University Digital Commons

Pharmacy Faculty Articles and Research

School of Pharmacy

2-16-2016

\title{
Development and Validation of a Sensitive UPLC- MS/MS Method for the Quantitation of [13C]Sucrose in Rat Plasma, Blood, and Brain: Its Application to the Measurement of Blood-Brain Barrier Permeability
}

Mohammad K. Miah

Texas Tech University Health Sciences Center

Ulrich Bickel

Texas Tech University Health Sciences Center

Reza Mehvar

Chapman University, mehvar@chapman.edu

Follow this and additional works at: http://digitalcommons.chapman.edu/pharmacy_articles

Part of the Animals Commons, Cardiovascular System Commons, Medicinal-Pharmaceutical Chemistry Commons, Nervous System Commons, and the Pharmacy and Pharmaceutical Sciences Commons

\section{Recommended Citation}

Miah MK, Bickel U, Mehvar R. Development and validation of a sensitive UPLC-MS/MS method for the quantitation of $[13 \mathrm{C}]$ sucrose in rat plasma, blood, and brain: Its application to the measurement of blood-brain barrier permeability. J Chromatogr B. 2016;1015-1016:105-110. doi: 10.1016/j.jchromb.2016.02.017 


\section{Development and Validation of a Sensitive UPLC-MS/MS Method for the Quantitation of [13C]Sucrose in Rat Plasma, Blood, and Brain: Its Application to the Measurement of Blood-Brain Barrier Permeability}

\section{Comments}

NOTICE: this is the author's version of a work that was accepted for publication in Journal of Chromatography $B$. Changes resulting from the publishing process, such as peer review, editing, corrections, structural formatting, and other quality control mechanisms may not be reflected in this document. Changes may have been made to this work since it was submitted for publication. A definitive version was subsequently published in Journal of Chromatography B, volume 1015-1016, in 2016. DOI:10.1016/j.jchromb.2016.02.017

The Creative Commons license below applies only to this version of the article.

\section{Creative Commons License} (c) $($ ) $(9)$

This work is licensed under a Creative Commons Attribution-Noncommercial-No Derivative Works 4.0 License.

\section{Copyright}

Elsevier 


\section{Development and validation of a sensitive UPLC-MS/MS method for the quantitation of $\left[{ }^{13} \mathrm{C}\right]$ sucrose in rat plasma, blood, and brain: Its application to the measurement of blood-brain barrier permeability}

Mohammad K. Miah", Ulrich Bickel ${ }^{\mathrm{a}, \mathrm{b},{ }^{*},}$, Reza Mehvar, ${ }^{\mathrm{a}, \mathrm{b}, \mathrm{c}, *}$

${ }^{a}$ Department of Pharmaceutical Sciences, School of Pharmacy, Texas Tech University Health Sciences Center, Amarillo, Texas, USA

${ }^{\mathrm{b}}$ Center for Blood-Brain Barrier Research, School of Pharmacy, Texas Tech University Health Sciences Center, Amarillo, TX, USA

c Department of Biomedical and Pharmaceutical Sciences, Chapman University, School of Pharmacy, Irvine, CA, USA

${ }^{*}$ Corresponding author at: Department of Biomedical and Pharmaceutical Sciences, Rinker Health Science Campus, Chapman University, 9401 Jeronimo Road, Irvine, CA 92168, USA. Tel.: +1 714516 5490; fax: +1 714516 5481.; E-mail address: mehvar@chapman.edu.

* Corresponding author at: Department of Pharmaceutical Sciences, School of Pharmacy, Texas Tech University Health Sciences Center, Amarillo, Texas, 1300 S Coulter Drive, Amarillo, TX 79106, USA. Tel.: +1 806414 9236; fax: +1 806356 4034.; E-mail address:

Ulrich.Bickel@ttuhsc.edu. 


\section{ABSTRACT}

Accurate and reproducible measurement of blood-brain barrier (BBB) integrity is critical in the assessment of the pathophysiology of the central nervous system disorders and in monitoring therapeutic effects. The widely-used low molecular weight marker $\left[{ }^{14} \mathrm{C}\right]$ sucrose is non-specific in the absence of chromatographic separation. The purpose of this study was to develop and validate a sensitive and reproducible LC-MS/MS method for the analysis of stable isotopemodified $\left[{ }^{13} \mathrm{C}_{12}\right]$ sucrose in brain, plasma, and blood to determine BBB permeability to sucrose. After addition of internal standard (IS, $\left[{ }^{13} \mathrm{C}_{6}\right]$ sucrose), the marker and IS were recovered from diluted rat blood, plasma, and brain homogenate by protein precipitation using acetonitrile. The recovery of the marker and IS was almost quantitative (90-106\%) for all three matrices. The recovered samples were directly injected into an isocratic UPLC system with a run time of 6 min. Mass spectrometry was conducted using multiple reaction monitoring in negative mode. The method was linear $\left(r^{2} \geq 0.99\right)$ in the concentration ranges tested for the diluted blood and plasma (10-1000 $\mathrm{ng} / \mathrm{mL})$ and brain homogenate $(1-200 \mathrm{ng} / \mathrm{mL})$. The lower limit of quantitation of the assay was $0.5 \mathrm{pg}$ injected on column. The assay was validated $(n=5)$ based on acceptable intra- and inter-run accuracy and precision values. The method was successfully used for the measurement of serial blood and plasma and terminal brain concentrations of $\left[{ }^{13} \mathrm{C}_{12}\right]$ sucrose after a single intravenous does $(10 \mathrm{mg} / \mathrm{kg})$ of the marker to rats. As expected, the apparent brain uptake clearance values of $\left[{ }^{13} \mathrm{C}_{12}\right]$ sucrose were low in healthy rats. The method may be useful for determination of the BBB integrity in animal models.

Keywords: Sucrose; Blood-brain barrier permeability; UPLC-MS/MS; Blood; Plasma; Brain 


\section{Introduction}

Measurement of the permeability of cellular barriers, in particular of vascular barriers, requires the use of suitable markers. If the goal is to determine passive, diffusion mediated permeability to small, drug like compounds, the choices are quite limited, because many small molecules are substrates of one or more of a host of facilitative or active transport systems [1]. A permeability of particular importance is the blood-brain barrier (BBB). The BBB is the microvascular barrier that strictly controls the passage of endogenous and exogenous molecules from blood to the central nervous system (CNS). It is formed by the endothelial cells of brain microvessels, which feature complex tight junctions resulting in extremely low paracellular permeability $[2,3]$. Many acute and chronic diseases affecting the CNS are accompanied by a compromised BBB [4]. Therefore, techniques to accurately and reproducibly measure BBB integrity are critical tools in the assessment of the pathophysiology of CNS disorders and in monitoring therapeutic effects.

The functional integrity of the BBB with respect to paracellular leakage is measured by low molecular weight hydrophilic compounds or by macromolecular markers. Most of the toxic substances that are believed to be associated with neurological complications (endogenous metabolites including ammonia, bile acids, creatinine, and glutamine, and xenobiotics including drugs) have molecular weights less than 1000 Da. Hence, low molecular weight markers of BBB permeability are of particular interest. Notwithstanding, the most frequently used marker in the BBB literature, the azo dye Evans blue (961 Da) [5], supposedly measures permeability to macromolecules due to its tight binding to serum albumin. Evidence for binding to multiple proteins other than albumin [6], and for potentially significant fractions of unbound dye [7] raises doubts about the soundness of Evans blue based data. Another common small molecule 
dye is fluorescein (376 Da). That marker may be prone to artifacts, too, since it can interact with efflux transporters present at the BBB [8]. Additionally, plasma protein binding of fluorescein must not be neglected. We recently reported that the free fraction of fluorescein is significantly increased by bile acids [9], leading to altered brain disposition of the marker in rat models of Pringle maneuver and partial hepatectomy [10]. Changes in the free fraction of fluorescein resulted in increased concentrations of fluorescein in the brain, without any significant changes in actual BBB permeability [10].

Unlike fluorescein, plasma protein binding or affinity to any known mammalian transporter does not affect the disaccharide sucrose (342 Da). It has long been established as a reliable marker of extracellular fluid volume [11, 12]. Since initial applications in the 1960s and 1970s [13-15], radiolabeled sucrose (e.g., $\left[{ }^{14} \mathrm{C}\right]$ sucrose) is considered a reliable low molecular weight, hydrophilic, poorly diffusible tracer for quantitative analysis of BBB integrity and has been used in more than 500 publications [5]. However, radiotracer use is associated with special handling and licensing requirements. Furthermore, with few exceptions radioactivity measurement is performed on samples without prior chromatographic separation and is thus nonspecific. Even small impurities in the dosing solution might significantly impact the outcome of such experiments $[16,17]$.

We propose that the advancement of new analytical methods, such as LC-MS/MS, should enable the use of non-radioactive sucrose as marker of BBB permeability. In fact, tandem mass spectrometry methods for measurement in body fluids of various sugars, including sucrose, have recently been described [18-20]. It is obvious, however, that sucrose from dietary sources is present in plasma of humans and animals at variable concentrations in the low micromolar range $[18,21]$. As a consequence natural $\left[{ }^{12} \mathrm{C}\right]$ sucrose cannot easily be utilized as a marker for the 
measurement of BBB permeability. Therefore, the purpose of the current study was to develop a sensitive and reproducible LC-MS/MS method for the analysis of stable isotope-modified $\left[{ }^{13} \mathrm{C}\right]$ sucrose in brain, plasma, and blood.

\section{Materials and methods}

\subsection{Chemicals and reagents}

We purchased $\left[{ }^{13} \mathrm{C}_{12}\right]$ sucrose (all the carbons in both glucose and fructose molecules are labeled with ${ }^{13} \mathrm{C}$ isotope; denoted ${ }^{13} \mathrm{C}$-sucrose) and the internal standard (IS), which was $\left[{ }^{13} \mathrm{C}_{6}\right]$ sucrose (all the carbons in fructose molecule are labeled with ${ }^{13} \mathrm{C}$ isotope), from Omicron Biochemicals (South Hill Street South Bend, IN, USA). LC-MS grade water was purchased under the brand name J.T. Baker from Avantor Performance Materials, Inc. (Center Valley, PA). Analytical grade ammonium hydroxide and LC-MS grade acetonitrile were purchased from Fisher Scientific (Fair Lawn, NJ). For anesthesia, ketamine and xylazine solutions were purchased from Lloyd Laboratories (Shenandoah, IA, USA). Heparin solution was purchased from APP Pharmaceuticals (Schaumburg, IL, USA). All other chemicals were UHPLC grade and obtained from commercial sources.

\subsection{Instrumentation}

For mass spectrometry, we used an $\mathrm{AB}$ SCIEX QTRAP ${ }^{\circledR} 5500$ mass spectrometer (Foster City, CA, USA). Liquid chromatography was performed using a Nexera UPLC system from Shimadzu Corporation (Columbia, MD). The UPLC system consisted of Sil-30AC autosampler, LC-30AD pumps, a CBM-20A controller, a DGA-20A5 degasser, and a CTO-30A column oven. For the acquisition of data and quantification, we used Analyst and Multi-Quant software, respectively. Chromatographic separation was performed using an Acquity BEH amide 
column with $3 \mathrm{~mm} \times 50 \mathrm{~mm}$ dimensions and a $1.7 \mu \mathrm{m}$ particle size, attached to an inline filter with a pore size of $0.2 \mu \mathrm{m}$ as a pre-column The isocratic mobile phase was acetonitrile: water: ammonium hydroxide $(72: 28: 0.1), \mathrm{v} / \mathrm{v})$, run at a flow rate of $0.2 \mathrm{~mL} / \mathrm{min}$. Column temperature was maintained at $45^{\circ} \mathrm{C}$, and the autosampler was at room temperature. The total run time was 6 min. However MS data were collected from 1.5 to $5 \mathrm{~min}$ only, and the valve was diverted to waste before and after that time.

\subsection{Mass spectroscopy conditions}

Analysis was performed by using a triple quadruple mass spectrometer to monitor the $\mathrm{m} / \mathrm{z}$ transitions with the help of the Analyst software connected to the system. The ionization source was electrospray ionization with multiple reactions monitoring system in negative mode. The mass spectrometer conditions were optimized to get optimum $\mathrm{M}-\mathrm{H}^{-1}$ by continuous injection of the standard solution with injection pump at a concentration of $100 \mathrm{ng} / \mathrm{mL}$ in a mixture of acetonitrile and water (80:20). The optimized mass spectrometer parameters were as follows: ion spray voltage, $-4500 \mathrm{~V}$; collision gas, high; curtain gas, $30 \mathrm{psi}$; temperature, $600{ }^{\circ} \mathrm{C}$; ion source gas 1 (nebulizer gas), $55 \mathrm{psi}$; and ion source gas 2 (turbo gas), 55 psi. The optimum transitions for ${ }^{13} \mathrm{C}$-sucrose and IS were $353 \rightarrow 92 \mathrm{~m} / \mathrm{z}$ and $347 \rightarrow 89 \mathrm{~m} / \mathrm{z}$, respectively.

\subsection{Standard curve preparation}

Stock solutions of ${ }^{13} \mathrm{C}$-sucrose as well as IS were made in water at a concentration of 10 $\mathrm{mg} / \mathrm{mL}$. Samples for standard curves were made in blank rat plasma, blood, or brain matrices. For plasma and blood standard curves, blank rat plasma or blood was spiked with the ${ }^{13} \mathrm{C}$-sucrose stock solutions in water to give concentrations of $1,2,5,10,25,50$, and $100 \mu \mathrm{g} / \mathrm{mL}$ for plasma and $0.5,1,2,5,10,25$, and $50 \mu \mathrm{g} / \mathrm{mL}$ for blood. The plasma and blood calibration standards 
were then diluted 100 and 50 fold with water to produce concentrations of $10,20,50,100,250$, 500 , and $1000 \mathrm{ng} / \mathrm{mL}$ before being subjected to the sample preparation procedure detailed below. The brain standards were prepared in blank brain homogenate, which was prepared by homogenization of brain in water (1:9). Brain homogenates were spiked with the stock solution of ${ }^{13} \mathrm{C}$-sucrose to give concentrations of $1,2,4,10,50,100$, and of $200 \mathrm{ng} / \mathrm{mL}$. Calibration curves were constructed by plotting the ratio of peak areas for the analyte: IS versus the spiked concentration of the analyte.

\subsection{Sample preparation}

As mentioned above, plasma and blood calibration standards and unknown samples were diluted 100 and 50 times, respectively, with water, and brain samples were homogenized in water (1:9) before analysis. Diluted plasma, blood, and undiluted brain homogenate samples were used for analysis. For sample preparation, $180 \mu \mathrm{L}$ of acetonitrile: water (80:20), containing 10 (for plasma or blood) or 2 (for brain) $\mathrm{ng} / \mathrm{mL}$ of IS, were added to $20 \mu \mathrm{L}$ of each sample in a microcentrifuge tube. Samples were then vortex-mixed for $15 \mathrm{sec}$, centrifuged at 20,000 g (room temperature) for $10 \mathrm{~min}$, and the supernatant was transferred into autosampler inserts for injection onto the column. The injection volume was $5 \mu \mathrm{L}$.

\subsection{Method validation}

The inter- and intra-run accuracies and precisions of the quality control samples were evaluated against calibration curves. For the calculation, five repeats of low, middle, and high concentrations were analyzed. The accuracy was calculated by measured concentration*100/nominal concentration. Precision was calculated as a percent of relative standard deviations (R.S.D.). The acceptable inter- and intra-run limits for the accuracy were set 
at $85-115 \%$ for the middle and high concentrations and $80-120 \%$ for the low concentration [22]. The acceptable precision values were 15\% (middle and high concentrations) and 20\% (low concentration) [22].

\subsection{Linearity}

The linearity of calibration curves was evaluated by the coefficient of determination $\left(r^{2}\right)$ of the linear regression analysis of the concentration-response data using a weight of $1 / x$, where $x$ is the concentration.

\subsection{Recovery}

The recovery of ${ }^{13} \mathrm{C}$-sucrose was calculated in diluted plasma and blood samples and in brain homogenates. Five repeats of two concentrations from the calibration curve, 50 and 500 $\mathrm{ng} / \mathrm{mL}$ for the plasma and blood or 10 and $100 \mathrm{ng} / \mathrm{mL}$ for the brain homogenate samples, were used. Samples with equivalent concentrations prepared in water (instead of matrix) were used as references. The samples and references were subjected to the Sample preparation method described above, and the peak areas of ${ }^{13} \mathrm{C}$-sucrose and IS were determined. Recovery was calculated by using the following equation:

Recovery $=\frac{\text { Peak Area of Sample }}{\text { Peak Area of Reference }} \times 100$

where Sample and Reference refer to the matrix and water, respectively.

\subsection{Application of the method}

The method was used to measure the plasma, blood, and brain concentrations of ${ }^{13} \mathrm{C}$ sucrose in rats after intravenous administration of the stable isotope. Additionally, the data were 
used to calculate the apparent brain uptake clearances $\left(K_{\text {in }}\right)$ of ${ }^{13} \mathrm{C}$-sucrose as a measure of BBB permeability [14]. The animal protocol used in this study was approved by our Institutional Animal Care and Use Committee (IACUC). Adult, male Sprague-Dawley rats were purchased from Charles River Laboratories, Inc. (Wilmington, MA, USA). Rats were kept in animal care facility in $12 \mathrm{~h}$ day/night cycles with free access to food and water for 2-3 days. After an overnight fast, rats $(n=3)$ were anesthetized with an intramuscular injection of ketamine: xylazine $(80: 8 \mathrm{mg} / \mathrm{kg})$, and catheters were placed in their penile vein and femoral artery for dosing and blood collection, respectively. ${ }^{13} \mathrm{C}$-sucrose, at a dose of $10 \mathrm{mg} / \mathrm{kg}$, was injected as a bolus dose into the penile vein, and serial blood samples were collected in heparinized microcentrifuge tubes at 0 (just before injection), 1, 5, 10, 15, 20, and $30 \mathrm{~min}$. An aliquot (20 $\mu \mathrm{L}$ ) of blood was kept for blood ${ }^{13} \mathrm{C}$-sucrose concentration measurement, and the remaining volume was centrifuged at room temperature for $5 \mathrm{~min}$ at a speed of $14,000 \mathrm{~g}$ to separate plasma. At the end of $30 \mathrm{~min}$, rat whole body was perfused with ice-cold saline for $5 \mathrm{~min}$ at a rate of 25 $\mathrm{mL} / \mathrm{min}$. Additionally, both jugular veins were cut to assure the lack of blood in the outflow solution, indicating complete washout of the residual blood in the brain. Brain was then collected and snap frozen in cold iso-pentane. Brain and water-diluted blood (50 fold) and plasma (100 fold) were kept at $-80^{\circ} \mathrm{C}$ until subjected to the analysis method described above. Apparent $K_{\text {in }}$ values were calculated using the following equations $[23,24]$ :

$$
\begin{gathered}
K_{\text {in }}^{\text {blood }}=\frac{C_{b r}^{30}}{A U C_{\text {blood }}^{0-30}} \\
K_{\text {in }}^{\text {plasma }}=\frac{C_{b r}^{30}}{A U C_{\text {plasma }}^{0-30}}
\end{gathered}
$$


where $K_{\text {in }}^{\text {blood }}$ and $K_{\text {in }}^{\text {plasma }}$ are the $K_{\text {in }}$ values based on the blood $\left(A U C_{b l o o d}^{0-30}\right)$ and plasma $\left(A U C_{\text {plasma }}^{0-30}\right)$ area under the sucrose concentration-time curves from time zero to $30 \mathrm{~min}$, respectively. The AUC values were determined by trapezoidal rule.

\section{Results}

\subsection{LC-MS/MS data}

Figure 1 depicts the chromatograms of brain, blood, and plasma matrices for blank samples (A), lowest ${ }^{13} \mathrm{C}$-sucrose standards in the calibration curves (B), and the lowest concentrations of ${ }^{13} \mathrm{C}$-sucrose observed in the samples at $30 \mathrm{~min}$ after a single $10 \mathrm{-mg} / \mathrm{kg}$ dose of ${ }^{13}$ C-sucrose (C). Also depicted in Fig. 1 are the chromatograms of IS (D). Under the chromatographic conditions used in our studies, the analyte $\left({ }^{13} \mathrm{C}\right.$-sucrose $)$ and IS eluted at 2.4-2.6 min as sharp peaks with low baseline noises without any interference from any of the matrices.

\subsection{Recovery}

Recoveries of ${ }^{13} \mathrm{C}$-sucrose and IS from the plasma, blood, and brain samples at a low and a high concentration in the calibration curves are presented in the Table 1 . The recoveries of ${ }^{13} \mathrm{C}$ sucrose and IS at both concentrations were relatively high $(\geq 90 \%)$, and in most cases quantitative, in all the tested matrices.

\subsection{Linearity}

Calibration curves constructed in the ranges of 10-1000 ng/mL (diluted plasma or blood) and $1-200 \mathrm{ng} / \mathrm{mL}$ (brain homogenate) were linear as the $r^{2}$ values were $\geq 0.99$ for all the matrices and the inter- and intra run calibration curves. The representative equations for the plasma, blood, and brain standard curves were $y=0.00820 x-0.00385\left(r^{2}=0.999\right), y=0.0125 x-$ 
$0.00475\left(r^{2}=0.998\right)$, and $y=0.0370 x-0.0165\left(r^{2}=0.998\right)$, where $y$ and $x$ refer to the ${ }^{13} \mathrm{C}$ sucrose: IS peak area ratios and the concentrations of ${ }^{13} \mathrm{C}$-sucrose, respectively.

\subsection{Inter- and intra-run accuracy and precision}

Table 2 shows the summary of accuracy and precisions values. The inter- and intra-run accuracy and precision values were within the limits of the FDA guidelines for the method validation. The accuracy was within $94.7-106 \%$ for all the tested concentrations and matrices (Table 2). Additionally, samples precision values (R.S.D) were $\leq 11 \%$, for all the various concentrations and matrices (Table 2).

\subsection{LLOD and $L L O Q$}

As demonstrated in Table 2 and Fig. 1B, we were able to accurately and precisely quantify the lowest concentrations of ${ }^{13} \mathrm{C}$-sucrose in the calibration curves of blood or plasma (10 $\mathrm{ng} / \mathrm{mL})$ and brain homogenate $(1 \mathrm{ng} / \mathrm{mL})$. The brain homogenate concentration of $1 \mathrm{ng} / \mathrm{mL}$ is equivalent to injection of $0.5 \mathrm{pg}$ of the analyte on the column. Therefore, the LLOQ for the assay is $0.5 \mathrm{pg}$. However, based on a signal to noise ratio of $\geq 3$, the LLOD of the assay is $0.1 \mathrm{pg}$.

\subsection{Application of the method}

The developed method was applied to estimate the plasma, blood, and brain concentrations of ${ }^{13} \mathrm{C}$-sucrose after injection of a single intravenous bolus dose $(10 \mathrm{mg} / \mathrm{kg})$ of the marker to rats. The plasma and blood concentration-time profiles (A), plasma and blood AUC (B), brain concentrations (C), and apparent brain $K_{\text {in }}$ values (D) of ${ }^{13} \mathrm{C}$-sucrose in rats are presented in Fig. $2 .{ }^{13} \mathrm{C}$-sucrose showed a multiexponential decline in both blood and plasma (Fig. 2A), with blood AUCs comprising 62\% of the plasma AUCs (Fig. 2B). The average ( \pm SD) values of the terminal brain concentration of ${ }^{13} \mathrm{C}$-sucrose at $30 \mathrm{~min}$ was $43.4 \pm 3.03 \mathrm{ng} / \mathrm{g}$ (Fig. 
2C), resulting in the blood and plasma $K_{\text {in }}$ values of $0.058 \pm 0.004$ and $0.095 \pm 0.011 \mu \mathrm{L} /(\mathrm{min} . \mathrm{g})$, respectively (Fig. 2D).

\section{Discussion}

The results of our study indicate the following: (i) a specific LC-MS/MS method could be developed, which allows accurate quantification of $\left[{ }^{13} \mathrm{C}_{12}\right]$ sucrose in brain, plasma and blood samples; (ii) the measurement is highly sensitive and reproducible; (iii) the LC-MS/MS based method presents an alternative to techniques based on radiolabeled tracers for permeability measurements.

In a pilot series of detector experiments, we used electrospray ionization and negative ion detection mode to optimize the detection method and sensitivity of the assay. The mass spectrometer conditions were optimized by continuous injection of the standard solution with injection pump to get the optimum $\mathrm{M}-\mathrm{H}^{-1}$. In the continuous scanning mode, most robust $\mathrm{m} / \mathrm{z}$ values for $\left[{ }^{13} \mathrm{C}_{12}\right]$ sucrose were found at 92,61 , and 89 . However, after running with mobile phase and column, the best signal to noise ratio was obtained at a $\mathrm{m} / \mathrm{z}$ of 92 . Therefore, the $\mathrm{m} / \mathrm{z}$ transition of $353 \rightarrow 92$ was selected for our LC-MS/MS analysis. Additionally, the selected transition for $\left[{ }^{13} \mathrm{C}_{6}\right]$ sucrose (IS) was $347 \rightarrow 89$.

In our studies, we used a BEH amide column for the chromatographic separation, which gives highly efficient separation of carbohydrates $[25,26]$. We used an isocratic condition, at a flow rate of $0.2 \mathrm{~mL} / \mathrm{min}$, which resulted in the elution of sucrose at $\sim 2.5$ min retention time after a $5 \mu \mathrm{L}$ sample injection volume. Our optimization studies indicated that the selection of mobile phase is very important as higher contents of water in the mobile phase caused a significant decrease in the sensitivity and much broader peaks. The optimum sensitivity and retention time 
were obtained with $0.1 \%$ ammonium hydroxide in a mixture of acetonitrile and water $(72: 28)$ (Fig. 1).

Recovery of the analyte depends on the procedure used to clean up the samples from the respective matrices. Deproteinization is one of the efficient methods for separation of low molecular weight hydrophilic compounds, where acetonitrile or other organic solvents are used alone or in combination with water [27]. We found a mixture of acetonitrile and water (80:20) for protein precipitation to get satisfactory recovery $(\geq 90 \%)$ from brain, plasma, and blood (Table 1). In contrast, the use of $100 \%$ acetonitrile for protein precipitation resulted in significantly lower sucrose recovery from the samples.

Previous reports also used a mixture of acetonitrile: water for precipitation of plasma proteins in the assay of natural sucrose in the serum of bottlenose dolphins [20] and horses [18]. However, both of these methods used an acetonitrile: water ratio of 90:10, instead of 80:20 used in our assay. Although the use of 90:10 ratio resulted in appropriate recoveries for the plasma and blood samples, the recovery was not quantitative for the brain homogenate in our studies.

We also examined the effects of the ratio of water to brain tissue during the homogenization of the brain on the recovery of the analyte and IS and/or brain matrix effect. As demonstrated in Table 2, using a ratio of 9:1 (water: brain tissue) and an acetonitrile: water ratio of 80:20 for protein precipitation resulted in a quantitative recovery of the analyte and IS from the brain without any matrix effect. However, lower water ratios for preparation of the brain homogenates resulted in lower recoveries and/or significant matrix effect (data not shown). The high and almost quantitative recovery of the analyte and IS from all matrices (Table 2) also is an indication of a lack of matrix effect using our sample preparation method. 
Applying the $\mathrm{m} / \mathrm{z}$ transitions outlined above, there was no substance peak in blank samples of blood, plasma, or brain tissue at the retention time of sucrose (Fig. 1A). Undetectable basal levels of $\left[{ }^{13} \mathrm{C}_{6}\right]$ sucrose and $\left[{ }^{13} \mathrm{C}_{12}\right]$ sucrose are expected in naïve samples due to the low natural isotopic abundance of ${ }^{13} \mathrm{C}$, which is $1.1 \%$. Therefore, natural sucrose contains $\left[{ }^{13} \mathrm{C}_{6}\right]$ sucrose and $\left[{ }^{13} \mathrm{C}_{12}\right]$ sucrose in fractions of about $1.8 \times 10^{-12}$ and $3.1 \times 10^{-24}$, respectively.

The developed LC-MS/MS method was successfully applied to measurements of the blood, plasma, and brain concentrations of ${ }^{13} \mathrm{C}$-sucrose after injection of the marker to rats at a dose of $10 \mathrm{mg} / \mathrm{kg}$. The biexponential decline in the blood or plasma concentrations of ${ }^{13} \mathrm{C}$ sucrose (Fig. 2 A) was similar to the profile previously reported for ${ }^{14} \mathrm{C}$-sucrose $[28,29]$. Additionally, the blood AUC was $62 \%$ of plasma AUC (Fig. 2B), which is in agreement with the lack of distribution of sucrose into red blood cells [11]. As expected from the ${ }^{14} \mathrm{C}$-sucrose data [15], the concentrations of ${ }^{13} \mathrm{C}$-sucrose in the brain were very low (Fig. 2C), relative to those in the blood or plasma (Fig. 2A), indicating the relative impermeability of the intact BBB to sucrose. The apparent brain uptake clearance of ${ }^{13} \mathrm{C}$-sucrose (Fig. 2D), was lower than that previously reported for ${ }^{14} \mathrm{C}$-sucrose $[14,15]$, which may be due to the lack of specificity of the radioactivity measurement for the latter. Indeed, previous studies [17] have shown that the $K_{i n}$ values obtained from ${ }^{14} \mathrm{C}$-sucrose is significantly affected by the degree of impurities in the injected ${ }^{14} \mathrm{C}$-sucrose.

\section{Conclusions}

In conclusion, a specific and sensitive LC-MS/MS method was developed for the measurement of ${ }^{13} \mathrm{C}$-sucrose in biological samples. The method was successfully applied to measurement of ${ }^{13} \mathrm{C}$-sucrose in blood, plasma, and brain after a single intravenous dose (10 
$\mathrm{mg} / \mathrm{kg}$ ) of the marker to rats. The method may be useful for determination of the blood-brain barrier integrity in normal and pathophysiologic conditions.

\section{Acknowledgements}

The authors would like to acknowledge financial support from the Blood-Brain Barrier Research Center at Texas Tech School of Pharmacy. Additionally, we would like to thank Dr. Helen Thorsheim for technical assistance during the development of this assay. 


\section{References}

[1] C.D. Klaassen, L.M. Aleksunes, Xenobiotic, bile acid, and cholesterol transporters: function and regulation, Pharmacol Rev, 62 (2010) 1-96.

[2] H.C. Bauer, I.A. Krizbai, H. Bauer, A. Traweger, "You Shall Not Pass"-tight junctions of the blood brain barrier, Front Neurosci, 8 (2014) 392.

[3] J.D. Huber, R.D. Egleton, T.P. Davis, Molecular physiology and pathophysiology of tight junctions in the blood-brain barrier, Trends Neurosci, 24 (2001) 719-725.

[4] R. Daneman, A. Prat, The blood-brain barrier, Cold Spring Harb Perspect Biol, 7 (2015) a020412.

[5] N.R. Saunders, K.M. Dziegielewska, K. Mollgard, M.D. Habgood, Markers for blood-brain barrier integrity: how appropriate is Evans blue in the twenty-first century and what are the alternatives?, Frontiers in neuroscience, 9 (2015) 385.

[6] M. Emmett, C.E. Cerniglia, A.J. Crowle, Differential serum protein binding of benzidineand benzidine-congener based dyes and their derivatives, Arch Toxicol, 57 (1985) 130135.

[7] V. Lindner, H. Heinle, Binding properties of circulating Evans blue in rabbits as determined by disc electrophoresis, Atherosclerosis, 43 (1982) 417-422.

[8] H. Sun, D.W. Miller, W.F. Elmquist, Effect of probenecid on fluorescein transport in the central nervous system using in vitro and in vivo models, Pharm Res, 18 (2001) 15421549.

[9] I.H. Shaik, M.K. Miah, U. Bickel, R. Mehvar, Effects of short-term portacaval anastomosis on the peripheral and brain disposition of the blood-brain barrier permeability marker sodium fluorescein in rats, Brain Res, 1531 (2013) 84-93. 
[10] M.K. Miah, I.H. Shaik, U. Bickel, R. Mehvar, Effects of Pringle maneuver and partial hepatectomy on the pharmacokinetics and blood-brain barrier permeability of sodium fluorescein in rats, Brain Res, 1618 (2015) 249-260.

[11] N.M. Keith, M.H. Power, The urinary excretion of sucrose and its distribution in the blood after intravenous injection into normal men, Am J Physiol, 120 (1937) 203-211.

[12] N. Deane, G.E. Schreiner, J.S. Robertson, The velocity of distribution of sucrose between plasma and interstitial fluid, with reference to the use of sucrose for the measurement or extracellular fluid in man, J Clin Invest, 30 (1951) 1463-1468.

[13] D.J. Reed, D.M. Woodbury, Kinetics of Movement of Iodide, Sucrose, Inulin and RadioIodinated Serum Albumin in the Central Nervous System and Cerebrospinal Fluid of the Rat, J Physiol, 169 (1963) 816-850.

[14] R.K. Ferguson, D.M. Woodbury, Penetration of 14C-inulin and 14C-sucrose into brain, cerebrospinal fluid, and skeletal muscle of developing rats, Experimental brain research, 7 (1969) 181-194.

[15] K. Ohno, K.D. Pettigrew, S.I. Rapoport, Lower limits of cerebrovascular permeability to nonelectrolytes in the conscious rat, Am J Physiol, 235 (1978) H299-307.

[16] E. Preston, J. Webster, Differential passage of [14C]sucrose and $[3 \mathrm{H}]$ inulin across rat blood-brain barrier after cerebral ischemia, Acta Neuropathologica, 103 (2002) 237-242.

[17] E. Preston, D.O. Foster, P.A. Mills, Effects of radiochemical impurities on measurements of transfer constants for [14C]sucrose permeation of normal and injured blood-brain barrier of rats, Brain Research Bulletin, 45 (1998) 111-116. 
[18] E. D'Arcy-Moskwa, L. Weston, G.N. Noble, S.L. Raidal, Determination of sucrose in equine serum using liquid chromatography-mass spectrometry (LC/MS), J. Chromatogr. B, 879 (2011) 3668-3671.

[19] P. Kubica, A. Kot-Wasik, A. Wasik, J. Namiesnik, P. Landowski, Modern approach for determination of lactulose, mannitol and sucrose in human urine using HPLC-MS/MS for the studies of intestinal and upper digestive tract permeability, J. Chromatogr. B, 907 (2012) 34-40.

[20] K.K. Buddington, W.E. Holmes, C.L. Clemons-Chevis, M.A. Solangi, D. Vanderpool, R.K. Buddington, Oral administration of sucrose solutions and measurement of serum sucrose concentrations to evaluate gastric permeability in adult bottlenose dolphins (Tursiops truncatus), Am J Vet Res, 67 (2006) 931-935.

[21] K. van Wijck, H.M. van Eijk, W.A. Buurman, C.H. Dejong, K. Lenaerts, Novel analytical approach to a multi-sugar whole gut permeability assay, J. Chromatogr. B, 879 (2011) 2794-2801.

[22] D. Zimmer, New US FDA draft guidance on bioanalytical method validation versus current FDA and EMA guidelines: chromatographic methods and ISR, Bioanalysis, 6 (2014) 1319.

[23] U. Bickel, How to measure drug transport across the blood-brain barrier, NeuroRx, 2 (2005) $15-26$.

[24] K. Ohno, K.D. Pettigrew, S.I. Rapoport, Lower limits of cerebrovascular permeability to nonelectrolytes in the conscious rat, Am. J. Physiol., 235 (1978) H299-H307.

[25] A.A. Ghfar, S.M. Wabaidur, A.Y. Ahmed, Z.A. Alothman, M.R. Khan, N.H. Al-Shaalan, Simultaneous determination of monosaccharides and oligosaccharides in dates using 
liquid chromatography-electrospray ionization mass spectrometry, Food Chem, 176 (2015) 487-492.

[26] Y. Liu, S. Urgaonkar, J.G. Verkade, D.W. Armstrong, Separation and characterization of underivatized oligosaccharides using liquid chromatography and liquid chromatographyelectrospray ionization mass spectrometry, J Chromatogr A, 1079 (2005) 146-152.

[27] M.K. Miah, I.H. Shaik, U. Bickel, R. Mehvar, Effects of hepatic ischemia-reperfusion injury on the P-glycoprotein activity at the liver canalicular membrane and blood-brain barrier determined by in vivo administration of rhodamine 123 in rats, Pharm Res, 31 (2014) 861873.

[28] M. Vavra, M.J. Ali, E.W. Kang, Y. Navalitloha, A. Ebert, C.V. Allen, D.R. Groothuis, Comparative pharmacokinetics of $14 \mathrm{C}$-sucrose in RG-2 rat gliomas after intravenous and convection-enhanced delivery, Neuro Oncol, 6 (2004) 104-112.

[29] E. Preston, N. Haas, M. Allen, Reduced permeation of 14C-sucrose, 3H-mannitol and 3Hinulin across blood-brain barrier in nephrectomized rats, Brain Res Bull, 12 (1984) 133136. 


\section{Table1}

Recoveries (mean $\pm \mathrm{SD}$ ) of ${ }^{13} \mathrm{C}$-sucrose and internal standard from plasma, blood, and brain samples at low and high concentrations in their respective calibration curves $(n=5)$.

\begin{tabular}{clc}
\hline $\begin{array}{l}\text { Concentration } \\
(\mathrm{ng} / \mathrm{mL})\end{array}$ & $\begin{array}{l}\text { Recovery (\%) } \\
{ }^{13} \mathrm{C}-\text { Sucrose }\end{array}$ \\
\hline Plasma & $98.8 \pm 0.5$ & IS \\
50 & $106 \pm 4.7$ & $90.0 \pm 0.8$ \\
500 & $100 \pm 2$ & $98.7 \pm 1.3$ \\
Blood & $105 \pm 2$ & $94.1 \pm 0.8$ \\
50 & & $101 \pm 2.5$ \\
500 & & \\
Brain Homogenate & $94.0 \pm 2.2$ & $97.7 \pm 2.5$ \\
10 & $100 \pm 1$ & $96.3 \pm 2.6$ \\
100 & & \\
\hline
\end{tabular}




\section{Table 2}

Intra- and inter-run accuracy and precision values of the lowest, middle, and highest concentrations of ${ }^{13} \mathrm{C}$-sucrose in standard curves for plasma, blood, and brain $(n=5)$.

\begin{tabular}{|c|c|c|c|c|}
\hline \multirow{2}{*}{$\begin{array}{l}\text { Concentration } \\
(\mathrm{ng} / \mathrm{mL})\end{array}$} & \multicolumn{2}{|l|}{ Intra-run } & \multicolumn{2}{|l|}{ Inter-run } \\
\hline & Accuracy & R.S.D. (\%) & Accuracy & R.S.D. (\%) \\
\hline \multicolumn{5}{|l|}{ Diluted plasma } \\
\hline 10 & 94.7 & 2.8 & 99.9 & 6.1 \\
\hline 100 & 104 & 3.1 & 103 & 1.9 \\
\hline 1000 & 102 & 1.8 & 98.1 & 2.5 \\
\hline \multicolumn{5}{|l|}{ Diluted blood } \\
\hline 10 & 98.6 & 2.9 & 104 & 8.2 \\
\hline 100 & 101 & 3.3 & 95.4 & 2.0 \\
\hline 1000 & 102 & 2.1 & 97.3 & 2.6 \\
\hline \multicolumn{5}{|c|}{ Brain homogenate } \\
\hline 1 & 106 & 3.9 & 99.7 & 10.2 \\
\hline 20 & 105 & 1.3 & 98.5 & 7.0 \\
\hline 200 & 95.8 & 2.6 & 98.0 & 2.8 \\
\hline
\end{tabular}




\section{Legend for Figures}

Fig. 1. Representative chromatograms of blank (A), lowest ${ }^{13} \mathrm{C}$-sucrose concentration in the calibration curves (B), ${ }^{13} \mathrm{C}$-sucrose in rat samples at $30 \mathrm{~min}$ after a single $10 \mathrm{mg} / \mathrm{kg}$ dose of the marker (C), and internal standard (D). Left, middle, and right panels represent brain, blood, and plasma chromatograms, respectively. The lowest standard curve sample for the brain contained 1 $\mathrm{ng} / \mathrm{mL}$ and for the blood and plasma contained $10 \mathrm{ng} / \mathrm{mL}$ of ${ }^{13} \mathrm{C}$-sucrose.

Fig. 2. Plasma and blood concentration time profiles (A), plasma and blood AUCs (B), brain concentrations (C), and apparent $K_{\text {in }}$ values (D) of ${ }^{13} \mathrm{C}$-sucrose. Rats $(n=3)$ were injected intravenously with $10 \mathrm{mg} / \mathrm{kg}$ of the marker, and serial blood/plasma samples were collected for $30 \mathrm{~min}$. Additionally, brain was collected at $30 \mathrm{~min}$. 
Brain

\section{A (Blank)}

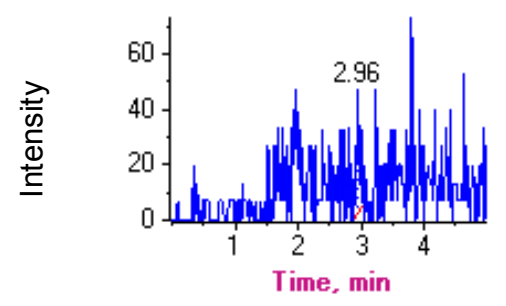

\section{B (Lowest Calibration Standards)}

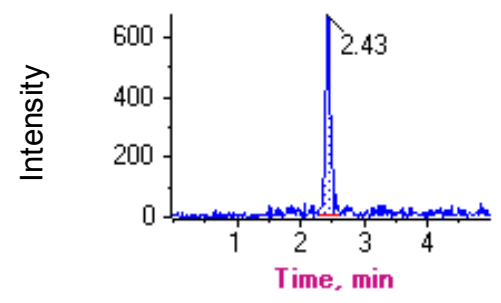

\section{C (Rat Samples at 30 Minutes)}

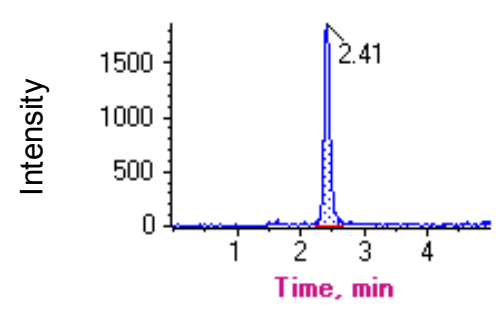

\section{D (Internal Standard)}

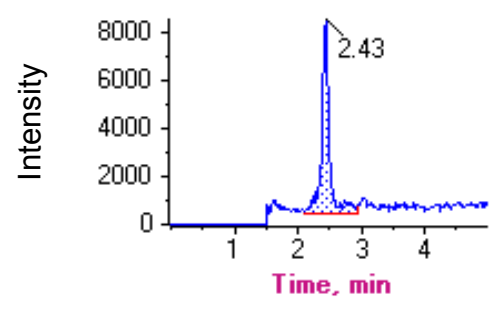

Blood
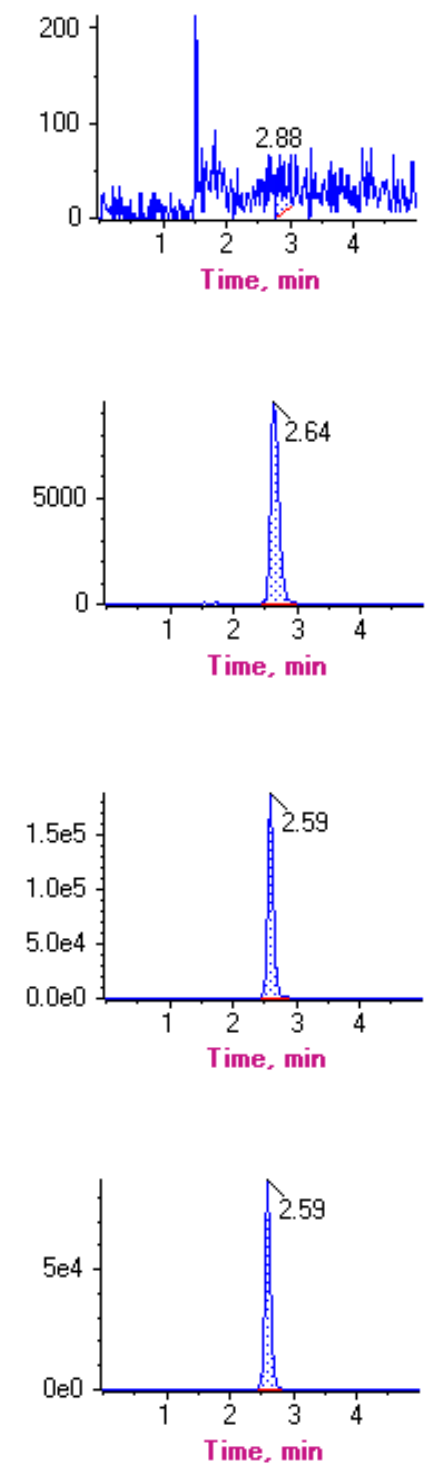

Plasma
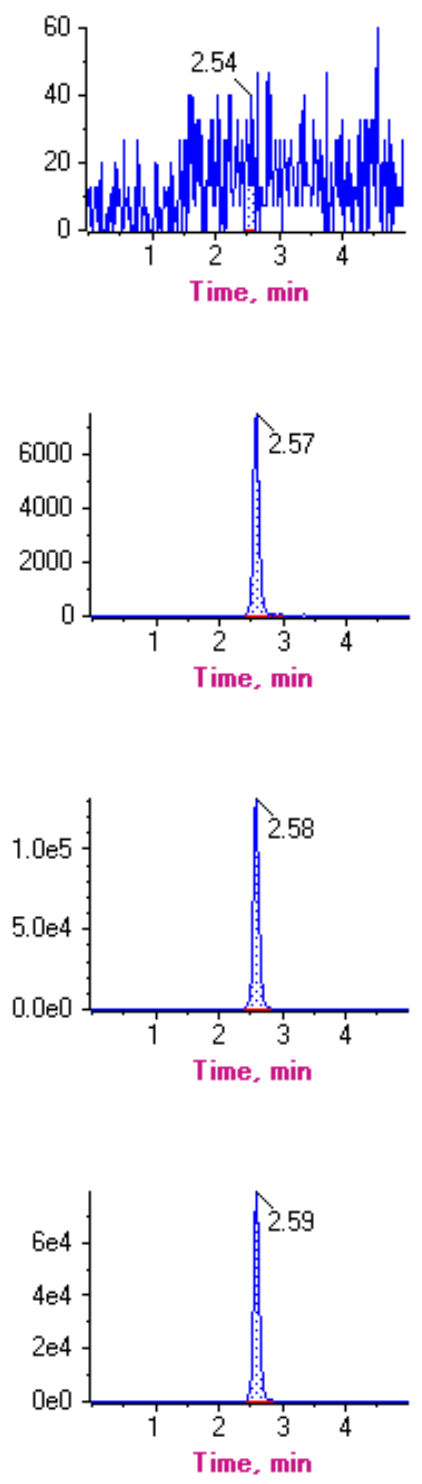
A

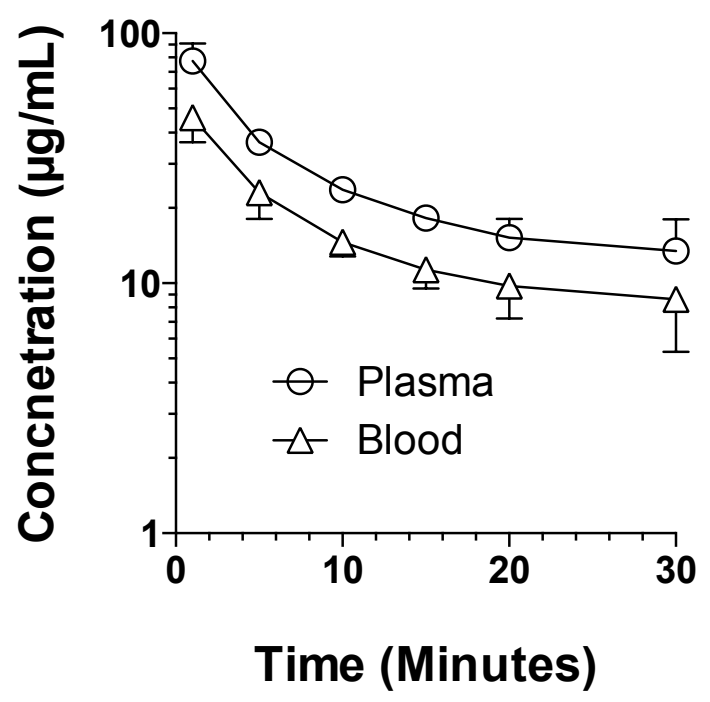

C

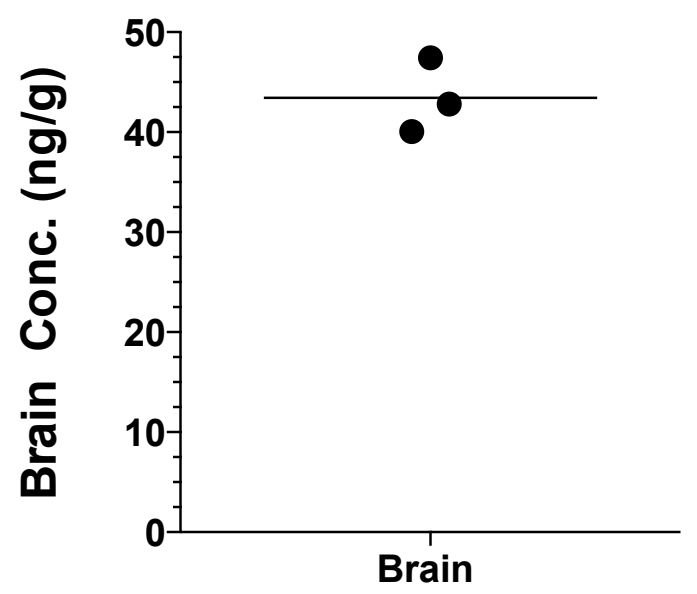

B

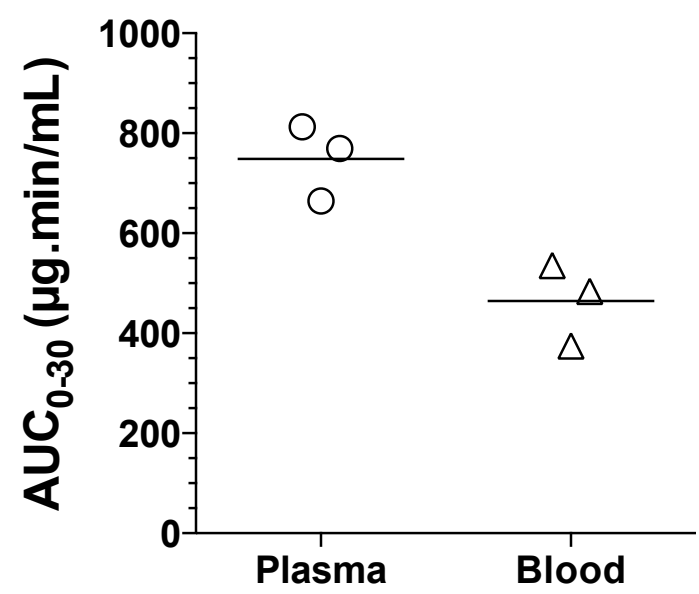

D

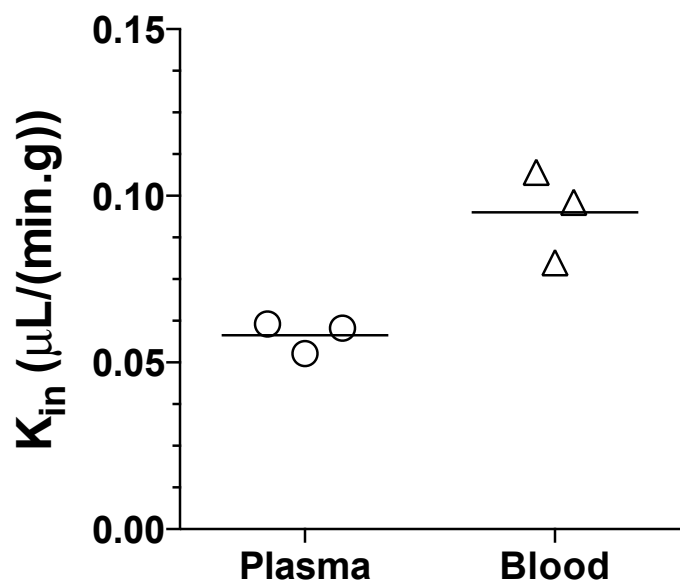

